\title{
Local Linear Approximation for Kernel Methods: The Railway Kernel
}

\author{
Alberto Muñoz ${ }^{1}$, Javier González ${ }^{1}$, and Isaac Martín de Diego ${ }^{2}$ \\ ${ }^{1}$ University Carlos III de Madrid, c/ Madrid 126, 28903 Getafe, Spain \\ \{alberto.munoz, javier.gonzalez\}@uc3m.es \\ ${ }^{2}$ University Rey Juan Carlos, c/ Tulipán s/n, 28933 Móstoles, Spain \\ isaac.martin@urjc.es
}

\begin{abstract}
In this paper we present a new kernel, the Railway Kernel, that works properly for general (nonlinear) classification problems, with the interesting property that acts locally as a linear kernel. In this way, we avoid potential problems due to the use of a general purpose kernel, like the RBF kernel, as the high dimension of the induced feature space. As a consequence, following our methodology the number of support vectors is much lower and, therefore, the generalizacion capability of the proposed kernel is higher than the obtained using RBF kernels. Experimental work is shown to support the theoretical issues.
\end{abstract}

\section{Introduction}

Support Vector Machines (SVM) have proven to be a successful method for the solution of a wide range of classification problems [1, 6]. In particular, linear SVMs are optimal in the classical setting in which two normally distributed populations have to be separated. This assertion is supported by the fact that SVM classifier approaches the optimal Bayes rule and its generalization error converges to the optimal Bayes risk 4]. Our aim in this paper is to build a global kernel for general nonlinear classification problems that locally behaves as a linear (optimal) kernel. Within this approach we expect to avoid the problems due to the use of a general purpose kernel like the RBF kernel: in this latter case, the data are embedded in a high dimensional feature space and problems of overfitting and poor generalization may appear. Since the proposed kernel behaves locally as a linear kernel, the good properties of the SVM classifier will be inherited by our method. In particular, the number of support vectors will be much lower and, therefore, the generalizacion capability will be higher than the obtained using RBF kernels.

To motivate our approximation, consider the situation presented in Figure 1 The decision function is clearly nonlinear. However, this function can be approximated locally by linear functions. For instance, a linear SVM (with kernel $K_{3}$ ) solves the classification problem in the oval area. We build a global kernel that will behave locally as the linear kernels whose decision functions are shown in the figure. We denote this kernel by 'Railway Kernel'. The name for this 


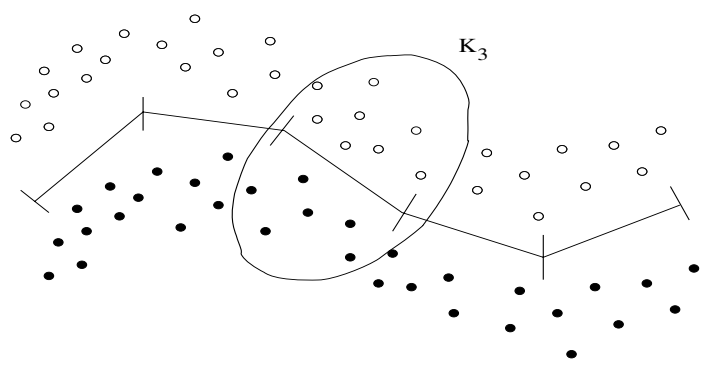

Fig. 1. Illustration of the Railway Kernel performance

kernel has been choosen because it is build like a railway where their wagons are the local decision functions.

The paper is organized as follows. The general framework for the proposed kernel is presented in Sections 2 and 3 . The experimental setup and results on various artificial and real data sets are described in Section 4 Section 5 concludes.

\section{Railway Kernel}

In this section we will study our new type of locally linear kernel, the Railway Kernel. We proceed as follows: First, the kernel is defined on 'simple' areas where the linear SVM works. Then the kernel is extended to the intersection of such 'pure' areas.

Next we introduce a special kernel that acts as an indicator function on the proccess.

\subsection{Indicator Kernel Functions}

Given a data set, let assume that we are able to identify specific space areas where the problem can be solved using a linear SVM. In this section we define a special indicator function to identify such areas. For the sake of simplicity only spherical areas are considered in this paper. The generalization to more elaborated shapes is straightforward. The indicator kernel function takes value 1 if the point under consideration is in the circular area defined by a given center and a radius, and decreases to zero quite fast as the distance to the center grows. Assumming smoothness in the boundary of the areas, we can define the following indicator kernel function $\lambda(x)$ :

$$
\lambda(x)=\left\{\begin{array}{ll}
1 & \text { if }\|x-c\|^{1 / 2} \leq r \\
e^{-\gamma\left(\|x-c\|^{2}-r^{2}\right)} & \text { if }\|x-c\|^{1 / 2}>r
\end{array} .\right.
$$

where $\|\cdot\|$ denotes the Euclidean distance, $x \in R^{d}$ is a sample point, $c \in R^{d}$ is the center of the sphere and $r>0$ is the radius. Parameter $\gamma>0$ is fixed in order to obtain a fast transition from 0 to 1 and, in this case, $\lambda(x)$ will aproximate 


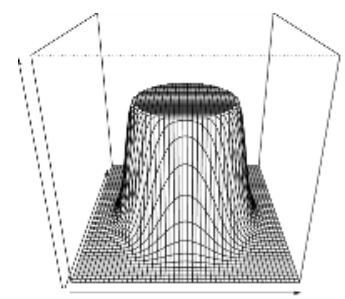

(a)

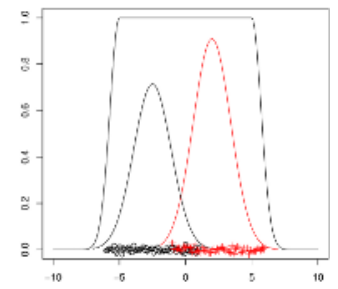

(b)

Fig. 2. Indicator kernel funcions. (a) 2D case. (b) 1D case for a two-class classification problem. Class density functions are shown.

an indicator function. It is immediate to check that $\lambda(x)$ is a kernel. The two dimensional case is shown in Figure 2a. Figure $2 \mathrm{~b}$ represents a two-class classification problem in one dimension and the corresponding indicator function. If a SVM kernel built from this indicator function is used to solve the classification problem, points outside the indicator influence will not be considered.

\subsection{Railway Kernel for a Two Areas Problem}

First consider the case of two areas without intersection. Kernel $K_{1}$ solves the classification problem in area $A_{1}$ and so does $K_{2}$ in area $A_{2}$. Let $x$ and $y$ be two sample data points. We define two functions: $H_{1}(x, y)=\lambda_{1}(x) \lambda_{1}(y)$ and $H_{2}(x, y)=\lambda_{2}(x) \lambda_{2}(y)$, where $\lambda_{1}$ and $\lambda_{2}$ are indicator kernel functions (with appropriate $c$ and $r$ parameters). The functions $H_{1}$ and $H_{2}$ take the value 1 when $x$ and $y$ belong to the same area, and 0 otherwise. In this particular case, we define the global Railway Kernel $K_{R}$ as follows:

$$
K_{R}(x, y)=H_{1}(x, y) K_{1}(x, y)+H_{2}(x, y) K_{2}(x, y) .
$$

Notice that the new kernel is obtained as a functional combination of linear kernels.

The Railway Kernel will approximate piecewise a global non-linear function by local linear functions. Notice that $K_{R}(x, y)$ is a block-diagonal matrix. This fact can be used to improve the optimization method used to solve the SVM problem (see [10] for details about the SVM optimization problem).

By the Representer Theorem (see [9] for details), the SVM solution takes the form: $f(x)=\sum_{i} \alpha_{i} K\left(x, x_{i}\right)+b$. In this case, due to the particular Railway Kernel structure the solution is given by:

$$
f(x)=\sum_{x_{i} \in A_{1}} \alpha_{i} K_{1}\left(x, x_{i}\right)+\sum_{x_{j} \in A_{2}} \alpha_{j} K_{2}\left(x, x_{j}\right)+b
$$

Notice that $K_{R}$ behaves like $K_{1}$ in the domain of indicator function $H_{1}$ and like $K_{2}$ in the domain of indicator function $\mathrm{H}_{2}$. 
We have not yet studied neither a multiarea problem, nor intersection between areas. These issues will be considered in Section 2.4

\subsection{A First Example}

We generate four groups of observations (50 observations per group) corresponding to four bivariate normal distributions: $N\left(\mu_{i}, \Sigma_{i}\right)$ for group $i$, with $\mu_{1}=(3,5), \mu_{2}=(7,5), \mu_{3}=(15,17), \mu_{4}=(15,13)$ respectively, and $\Sigma_{1}=\Sigma_{2}=$ $\operatorname{diag}(0.5,1.5)$ and $\Sigma_{3}=\Sigma_{4}=\operatorname{diag}(1.5,0.5)$. Points in groups 1 and 3 belong to class +1 and points in groups 2 and 4 belong to class -1 . Consider two areas defined by indicator kernel funcions with centers $c_{1}=(5,5), c_{2}=(15,15)$ and radii $r_{1}=r_{2}=5$ respectively. The point in this example is that the classes are linearly separable in each of these areas; however there is no a global proper linear kernel. In this case, the problem could be solved with a RBF kernel $(\sigma=1)$. Nevertheless when the Railway Kernel is used several advantages appear. The number of support vector is significatively lower than in the RBF case $(13.5 \%$ vs. $73.5 \%$ ). Figure 3 and 3 b show the decision functions for the Railway and RBF kernels respectively. In addition, the number of positive eigenvalues of the kernel

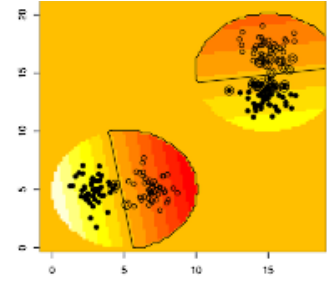

(a) Local linear kernels solution.

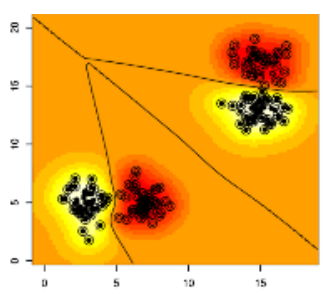

(b) Global RBF solution.

Fig. 3. Two solutions for a modified XOR problem (support vectors are highlighted)

matrix is clearly lower using the Railway Kernel (2.0\% vs. 25\%). Therefore, the manifold induced by the Railway Kernel is of lower dimension than the obtained using the RBF kernel. Figures 4 a and $4 \mathrm{~b}$ show the eigenvalues for the Railway and RBF kernels respectively.

\subsection{Building the Railway Kernel in the Intersections}

Next we deal with the problem of intersection between areas. Let $A_{1}$ and $A_{2}$ the areas under consideration. In this case, the Railway Kernel is built as follows:

$$
K_{R}(x, y)= \begin{cases}K_{1}(x, y) & \text { if } x, y \in A_{1} \cap A_{2}^{c}, \\ K_{2}(x, y) & \text { if } x, y \in A_{1}^{c} \cap A_{2}, \\ \frac{1}{2}\left(K_{1}(x, y)+K_{2}(x, y)\right) & \text { if } x, y \in A_{1} \cap A_{2}, \\ 0 & \text { otherwise, }\end{cases}
$$

where $A_{i}^{c}$ represents the complementary set of $A_{i}$. 


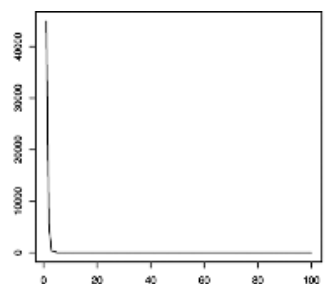

(a) Local linear kernel.

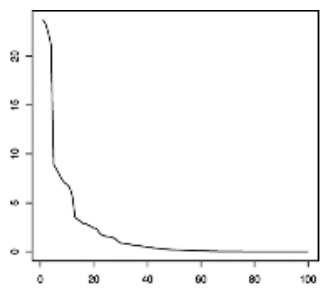

(b) RBF kernel matrix.

Fig. 4. Eigenvalues of the kernel matrices for the modified XOR problem

Intersections between areas can be seen as areas where both kernels achieve the same performance, and should be equally weighted. Thus, the average of the kernels (which is a kernel [2]) is computed for points in the intersection. Figure 5 a shows graphically the idea of intersection, and Figure $5 \mathrm{~b}$ shows the Railway Kernel perfomance in a simple example.

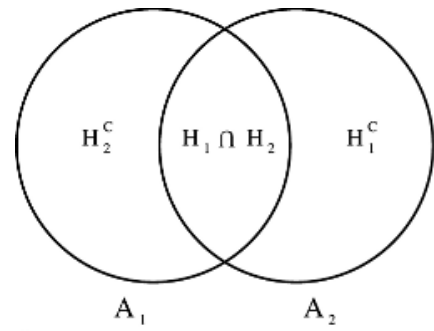

(a) Conceptual intersections in the Railway Kernel

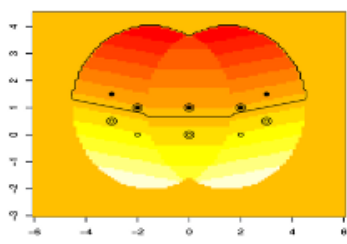

(b) Railway Kernel performance in a simple example with curvature.

Fig. 5. The Railway Kernel performance in an simple example with intersection

The matrix computed in (4) is a semidefinite positive and block diagonal matrix. Thus, it comes from a Mercer kernel. It is possible to find an analytical expression for (44). Consider the example given in Figure 5a). Without loss of generality suppose that our sample is distributed in 3 zones: $H_{1}^{c}, H_{2}^{c}$ and $H_{1} \cdot H_{2}$, where $H_{1}^{c}(x, y)$ is the region of the space where the funcion $H_{1}$ vanishes and it is given by $H_{1}^{c}=\left(1-\lambda_{1}(x)\right)\left(1-\lambda_{1}(y)\right)$. Thus, it represents those points in $A_{2}$ and not in $A_{1} . H_{2}^{c}$ represents those points in $A_{1}$ and not in $A_{2}$. The final kernel $\left(K_{R}\right)$ will be the sum of three matrices. $K_{R}(x, y)=0$ when $x$ and $y$ belong to different zones. In other case, $K_{R}(x, y)$ is exactly the kernel that works on the zone $x$ and $y$ belong to. The expression for the kernel is as follows:

$$
K_{R}(x, y)=H_{1}^{c}(x, y) K_{2}(x, y)+H_{2}^{c}(x, y) K_{1}(x, y)+
$$




$$
\left(H_{1}(x, y) H_{2}(x, y)\right) \frac{1}{2}\left(K_{1}(x, y)+K_{2}(x, y)\right) .
$$

As before, $K_{R}$ is a block-diagonal matrix where each block is a kernel matrix. Then, $K_{R}$ is a positive semidefinite matrix and thus a kernel. The generalization of (5) to the case of more than 2 areas is straightforward.

Notice that, to compute the Railway Kernel it is enough to use the areas information and the local linear kernels on that areas. We have built a method to compute an aproximation to a nonlinear decision function with a sum of local linear hiperplanes.

\section{Areas Location}

In Section 2 we have assumed that each point in the sample belongs to one or more previously defined areas. Now we present a local algorithm to detect such areas in a classification problem. The algorithm works in two main steps. First, single labelled areas are created. A sample point is assigned to its nearest area if the label of the point is the same that the label in the area. In other case, a new area is defined. A new area is built when the distance between the new point under consideration and its nearest area is higher than the maximun of the average distances into the areas. We repeat the process until each sample point belong to an area. Once the areas $A_{1}, \ldots, A_{M}$ have been built, the final areas are obtained joining the nearest areas with different labels. In order to obtain the indicator function kernels needed to build the Railway Kernels, centers and radii are needed. Centers are computed as the $A_{1}, \ldots, A_{M}$ centroids, and radii in each area are computed as the maximun distance between the center and the farthest point in this area.

An example to illustrate of the performance of the algorithm is presented in Figure 6. Figure 6a presents a two class problem in two dimensions. In Figure $6 \mathrm{~b}$ the result of applying the areas location algorithm is shown. The procedure is as follows. First, area 1 is created and completed. Next, we look for the next

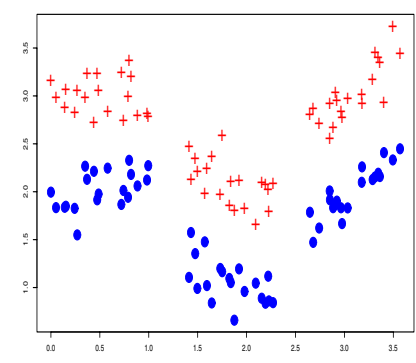

(a) Initial problem.

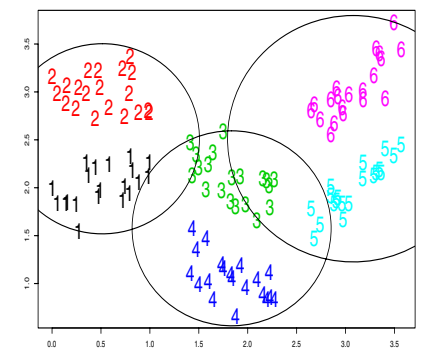

(b) Areas Location Solution.

Fig. 6. An example of the Areas Location algorithm performance 
nearest point. Since a data point belongs to another class a new area is defined (area 2). This new area is completed and a new point is evaluated. Since the distance between the previous area (2) and the nearest point (the new point) is large enough, a new area is created (area 3), and so on. Figure 6b shows the six final spherical areas detected.

\section{Experiments}

To test the performance of the proposed method, a SVM (with the upper bound on the dual variables fixed to 1) has been trained on artificial and real data sets using the Railway Kernel matrix previously constructed. We have compared the proposed methods with two SVM classifiers built using RBF kernels. For the first classifier $\left(\mathrm{SVM}_{1}\right)$ the parameter $\sigma$ is choosen as a function of the data dimension (see [8] and [7] for details). For the second $\left(\mathrm{SVM}_{2}\right), \sigma$ and the upper bound on the dual variables of the optimization problem are choosen following the ideas in 3 .

\subsection{Two Areas with Different Scattering Matrices}

The first data set under consideration is presented in Figure 7 and corresponds to 400 points in $\mathbb{R}^{2}$. There are two areas of points $(80 \%$ of the sample is in area $A_{1}$ and $20 \%$ is in area $\left.A_{2}\right)$. Each area $A_{i}$ corresponds to a normal cloud. The first area center is $(0,1)$ and the second group center is $(1,1)$, while the diagonal covariance matrices are $\sigma_{i}^{2} I$ where $\sigma_{1}=10^{-2} \sigma_{2}$, and $\sigma_{2}=1$. The point on this example is that the areas do not coincide with the classes $\{-1,+1\}$ that are to be learned. Half of the points in each class belongs to area $A_{1}$, and the other half to area $A_{2}$. Within each area, the classes are linearly separable. Therefore, the only way to build a proper classifier for this data set is to take into account the area each point belongs to. We use $50 \%$ of the data for training and $50 \%$ for testing.

To compare the performance of the Railway Kernel, consider a set of three RBF kernels with parameters $\sigma=0.5,5$ and 10 respectively.

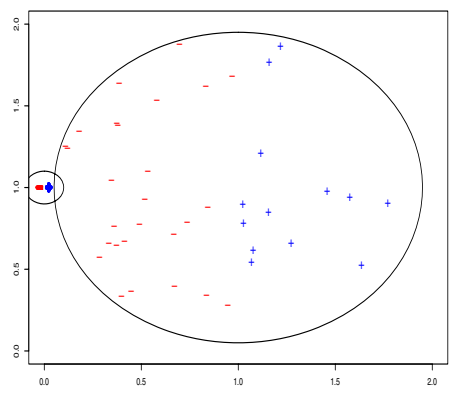

Fig. 7. Two areas with different scattering matrices. The first area center is $(0,1)$ and the second area center is $(1,1)$. The areas do not coincide with the classes $\{-1,+1\}$. 
Table 1 shows the performance of our proposal for this data set. The results have been averaged over 10 runs. Given the geometry of the data, it is clear that is not possible to choose a unique best $\sigma$ for the whole data set. As $\sigma$ grows, the test error increases for the data contained in area $A_{1}$, and decreases within area $A_{2}$. The Railway Kernel clearly improves the best RBF kernel.

Table 1. Percentage of missclassified data and percentage of support vectors for the two different scattering data set: $A_{1}$ stands for the less scaterring group, $A_{2}$ stands for the most dispersive one

\begin{tabular}{|c|c|c|c|c|c|}
\hline \multirow[b]{2}{*}{ Method } & \multicolumn{2}{|l|}{$\begin{array}{l}\text { Train } \\
\text { Error }\end{array}$} & \multicolumn{2}{|c|}{$\begin{array}{c}\text { Test } \\
\text { Error }\end{array}$} & \multirow{2}{*}{$\begin{array}{r}\text { Support } \\
\text { Vectors } \\
\text { Total }\end{array}$} \\
\hline & Total $A_{1}$ & $\overline{A_{2}}$ & Total & $A_{1} \quad A_{2}$ & \\
\hline $\mathbf{R B F}_{\sigma=0.5}$ & $2.4 \quad 3.0$ & 0.0 & 13.4 & 4.151 .0 & 39.2 \\
\hline $\mathbf{R B F}_{\sigma=5}$ & $4.6 \quad 5.8$ & 0.0 & 13.6 & 8.635 .0 & 82.6 \\
\hline $\mathbf{R B F}_{\sigma=10}$ & 29.136 .2 & 0.5 & 36.0 & 44.110 .0 & 94.4 \\
\hline Railway Kernel & $3.7 \quad 3.6$ & 15.6 & 4.2 & 0.120 .6 & 14.1 \\
\hline $\mathbf{S V M}_{1}$ & $2.1 \quad 2.6$ & 0.0 & 13.5 & 4.151 .0 & 39.6 \\
\hline $\mathbf{S V M}_{2}$ & $2.1 \quad 2.6$ & 0.0 & 11.0 & 3.341 .5 & 37.6 \\
\hline
\end{tabular}

\subsection{The Breast Cancer Data Set}

In this section we have dealt with a database from the UCI Machine Learning Repository: the Breast Cancer data set [5]. The data set consists of 683 observations with 9 features each. We use $80 \%$ of the data for training and $20 \%$ for testing.

Table 2 shows the performance of the Railway Kernel on this data set. Again, the results have been averaged over 10 runs. Our method clearly improve the RBF kernel with $\sigma$ parameter choosen as a function of the data dimension. Our method does not take into account the penalization parameter of the SVM. However, our results are similar to the classification results obtained when both parameters, $\sigma$ and the upper bound on the dual variables of the optimization problem, are choosen, but using significantly less support vectors.

Table 2. Percentage of missclassified data, sensitivity (Sens.), specificity (Spec.) and percentage of support vectors for the cancer data set. Standard deviations in brackets.

\begin{tabular}{|c|c|c|c|}
\hline \multirow[b]{2}{*}{ Method } & Train & Test & \multirow{2}{*}{$\begin{array}{l}\text { Support } \\
\text { Vectors }\end{array}$} \\
\hline & Error Sens. Spec. & Error Sens. Spec. & \\
\hline Railway Kernel & $2.5(0.3) 0.9790 .974$ & $2.9(0.4) 0.9750 .876$ & $18.6(3.6)$ \\
\hline $\mathbf{S V M}_{1}$ & $0.1(0.1) 1.0000 .999$ & 4.2 (1.4) 0.9890 .942 & $49.2(1.0)$ \\
\hline $\mathbf{S V M}_{2}$ & $0.0(0.0) 1.0000 .999$ & 2.9 (1.6) 0.9630 .975 & $49.2(1.0)$ \\
\hline
\end{tabular}




\section{Comments and Conclusions}

In this paper we have presented a new kernel, the Railway Kernel. This global kernel takes advantage of the good generalization properties of the local linear kernels for classification tasks. We have shown that the potential problems due to the use of a general purpose kernel like the RBF kernel have been avoid. The generalization capability of the proposed kernel is higher than the obtained using RBF kernels. The method could be generalized by using alternative nonlinear local kernel. Further research will focus on the theoretical properties of the Railway Kernel and extensions.

\section{References}

1. C. Cortes and V. Vapnik. Support Vector Networks. Machine Learning, 20:273-297, 1995.

2. N. Cristianini and J. Shawe-Taylor. An introduction to Support Vector Machine. Cambridge University Press, 2000.

3. S.S. Keerthi and C. Lin. Asymptotic Behaviors of Support Vector Machines with Gaussian Kernel. Neural Computation, 15:1667-1689, 2003.

4. Y. Lin., G. Wahba, H. Zhang and Y. Lee. Statistical Properties and Adaptive Tuning of Support Vector Machines. Machine Learning, 48:115-136, 2002.

5. O.L. Mangasarian and W.H. Wolberg. Cancer diagnosis via linear programming. SIAM News, 23 (5):1-18, 1990.

6. J. Moguerza and A. Muñoz. SVM with aplications. Statistical Science, In press, 2006.

7. A. Muñoz and J. M. Moguerza. Estimation of High-Density Regions Using One Class Neighbor Machines IEEE Transactions on Pattern Analysis and Machine Intelligence, 28 (3):476-480, 2006.

8. B. Schölkopf, J.C. Platt, J. Shawe-Taylor, A.J. Smola and R.C. Williamson. Estimating the Support of a High Dimensional Distribution. Neural Computation, 13(7):1443-1471, 2001.

9. B. Schölkopf, R. Herbrich, A. Smola and R. Williamson. A Generalized Representer Theorem. NeuroCOLT2 TR Series, NC2-TR2000-81, 2000.

10. B. Schölkopf and A. Smola. Learning with Kernels. MIT Press, 2002. 